\title{
Pervasive Internet Access by Vehicles through Satellite Receive-only Terminals
}

\author{
Baber Aslam, Ping Wang and Cliff C. Zou \\ School of Electrical Engineering and Computer Science \\ University of Central Florida \\ Orlando, FL, USA \\ \{ababer, pwang, czou\}@cs.ucf.edu
}

\begin{abstract}
Ubiquitous Internet connectivity is very important in present environment. A lot of research has been done to extend Internet connectivity to vehicular ad hoc networks (VANETs). The biggest challenge to achieve this goal is the requirement of pervasive fully networked roadside infrastructure. This requirement is difficult to achieve especially during the initial deployment phase of vehicular networks and also in areas with scarce roadside infrastructure (such as along highways and in rural areas). This makes solutions that are solely dependent on roadside infrastructure impracticable to be implemented. Other solutions using cellular networks or symmetric satellite communication are either expensive or do not provide sufficient bandwidth. Further satellite communication suffers heavy losses in urban area and makes its use difficult in vehicular networks.
\end{abstract}

We present a solution that complements the existing ones without requiring a fully networked roadside infrastructure. The solution uses satellite receive-only terminals and very few (widely spaced) roadside units to provide pervasive Internet connectivity. The solution is cost effective, incremental and practical. It can support TCP connection even when the uplink is interrupted for long durations of time. We present several different design options with varying degrees of error handling capabilities and different overheads and delays, which can be used according to the given environment.

Keywords VANET; asymmetric satellite; Internet; low penetration; initial deployment; Land mobile satellite communication

\section{INTRODUCTION}

Communication and especially the connectivity to the Internet is the basic requirement of most modern productive environments. We spend a considerable time traveling from one point to another via vehicles; this time can be more productive if we are connected to the Internet. A lot of research has been done to bring the Internet to vehicles. To this end, three main approaches have been adopted: Internet through roadside infrastructure alone or through roadside infrastructure using vehicle to vehicle (V2V) communication [1 - 5], Internet through cellular network $[1,6,7]$ and Internet through satellite (symmetric/asymmetric) [8, 9]. However, all three approaches have some challenges to deal with.

The Internet access through roadside infrastructure requires pervasive roadside units (RSUs) to achieve connectivity, since the typical radial range of an RSU is $250 \mathrm{~m}$ so we need an RSU every 400 to $500 \mathrm{~m}$. Further, these RSUs must all be connected to the Internet. Also, the installation, connection and maintenance of these RSUs will be quite expensive, and it may not be possible to achieve the desired connectivity, especially during the initial days of VANET deployment and along highways or in rural areas.

The Internet access through cellular networks, besides having smaller bandwidth, has more cost per bit of data as compared to other means. The cellular networks are designed considering statistical calling properties and these are quite different from the Internet usage patterns. Further, the cellular networks are symmetric and do not take advantage of Internet traffic's asymmetric property. Therefore, the cellular networks may not be able to handle huge volumes of data especially when the entire vehicle population in a cell area tries to access the Internet. In addition to these issues, when a vehicle crosses international boundary, the service providers and their carrier frequencies will often change, which makes user equipment more expensive and complex. Multiple service providers also mean multiple gateways and billing/licensing issues.

The Internet access through symmetric satellite requires vehicles to be equipped with satellite transceiver, which adds to the cost of user equipment. Satellite channel suffers heavily from losses; these losses introduce errors in the communication and require some error correction mechanisms. These losses are much more pronounced in urban areas where the areas are congested with buildings and other manmade objects. Therefore Internet access through satellite is particularly not an economical solution for urban areas.

The Internet and also some of the possible VANET applications exhibit asymmetric nature of traffic, in this downlink traffic is many orders of magnitude as compared to the uplink traffic [10]. This asymmetry is likely to increase with time as more and more content is becoming multimedia in nature. Satellite as downlink coupled with terrestrial lowbandwidth return channel (such as dial-up) to provide Internet to home users especially in rural areas has been used successfully for quite sometime $[11,12]$. Therefore, we use asymmetric satellite communication (downlink only). The use of satellite to provide connectivity in rural areas also seems logical since there will be less losses and hence low errors in rural areas (or along highways). The uplink is via roadside

This work was supported by NSF Cyber Trust Grant CNS-0627318 and Intel Research Fund. 
infrastructure using vehicle to infrastructure (V2I) communication or $\mathrm{V} 2 \mathrm{~V}$ in conjunction with V2I communication. However, the limited number of RSUs makes the traditional asymmetric satellite solutions impracticable. The challenge in VANET is the intermittent availability of terrestrial return channel with possible long disruption periods especially during the initial days of VANET deployment.

In this paper we present a solution that complements the existing ones and provides Internet connectivity during the initial deployment phase of the vehicular networks and also in areas with very scarce roadside infrastructure (such as along highways and in rural areas). The solution uses satellite receive-only terminals and very few (widely spaced) RSUs. The use of satellite receive-only terminals helps in keeping the user cost low. It can support TCP connection even when the uplink is interrupted for long durations of time. We present a number of options with varying degrees of error handling capabilities and recommend their usage according to the environment. Later on when additional RSUs are installed then the solution improves its performance by making more use of RSUs and also by reduction of inter-RSU distance. The solution is cost effective, incremental and practical.

A lot of research to address challenges of Internet (especially TCP performance) over delay tolerant networks (DTN)/satellite networks has been carried out. In order to avoid the repetitions, we will, in this paper, not focus on lower level details of what particular Internet protocol to be used; rather, we will identify the desired characteristics of the protocol and any already defined protocol (or combinations of these) can be used for the proposed solution.

The paper is organized in 5 sections. Section II discusses related research work in the field. Section III explains important characteristics of satellite communication and mobile satellite communication model. Section IV discusses the proposed system design and its various options along with the recommended usage. And in the end, section $\mathrm{V}$ presents conclusion and future work.

\section{RELATED WORK}

The solutions presented so far for provision of Internet to vehicles can be broadly divided into three categories; first, the solutions relying on roadside infrastructure or vehicle to vehicle communication, second, the solutions relying in some way on cellular networks and third, the solutions making use of satellite links. We will refer some of the important research papers in these categories.

A number of researches such as FleetNet, Drive-thru Internet, etc extensively rely on road side infrastructure and/or vehicle to vehicle communication to provide Internet connectivity to vehicles $[1,2,4]$. The basic requirement for these solutions is availability of pervasive roadside infrastructure and/or a large number of smart vehicles. Both these assumptions are not realistic during the initial deployment stage, further use of vehicle to vehicle communication has many security issues, such as privacy, confidentiality, denial of service etc. Solutions based on existing WiFi networks face similar problems [5].
A number of solutions incorporate cellular networks to provide Internet to the vehicles [1, 6, 7]. Cellular networks are mostly used as backbone; a replacement to roadside infrastructure. Cellular networks though pervasive have several disadvantages, such as, expensive to built/maintain, higher cost per bit of data, low data rates (especially at vehicular speed), heterogeneous technologies (WAP, GPRS, EDGE, HSDPA, etc [13]), billing/licensing issues among different service providers, higher roaming rates, large and variable latency, central switching/resource management, difficult to scale, occasional blackouts, etc [3, 4, 14-16].

Use of satellite channel for provision of Internet to terrestrial (static) and mobile users has been an interesting topic of research. Most of the researches in this area are related to performance-studies or enhancements of Internet protocols over symmetric/asymmetric satellite channels with stationary nodes $[11,12,17,18]$. There are also quite a few researches dealing with the mobile nodes but most of these study Internet protocol performance/enhancements [8, 9]. Further these consider symmetric satellite channel i.e., both uplink and downlink communication takes place via satellite. Symmetric communication requires expensive transceiver at the mobile nodes and it does not take advantage of the asymmetric nature of Internet communication. In this paper we are using satellite downlink communication only and the nodes are mobile nodes.

Our work comes closer to [19], where asymmetric satellite communication has been used for provision of Internet to the mobile nodes. In [19] the satellite is only used for downlink and uplink is via cellular network. The system design requires the mobile node to be equipped with both the satellite and cellular interfaces. The design suffers from the disadvantages of using cellular network (described above). Also, the design does not incorporate any roadside infrastructure, which when available could provide much higher data rates at lower costs. Further this also implies that the design will not be very successful in urban areas since satellite communication is not very reliable in urban areas (connection/fade ratio can be 33.3/66.6 in higher density cities like New York [20]).

Our system design differs in a number of ways from the researches presented above. First, we use satellite communication for downlink only thus reducing complexity of user terminals and operating costs. We use roadside infrastructure for uplink communication and do not need any cellular transceivers or satellite transmitters at nodes. This eases compatibility with other vehicular network architectures. The design works with very small number of RSUs and is especially suited for initial deployment stages. We present a number of options with varying degrees of error handling capabilities and recommend their suitability for different environments.

\section{SATEllite ChANNEL}

\section{A. Channel Characteristics}

The satellite channel is characterized by long delays, high fading/attenuation to signal, one way, high bandwidth and inorder packet delivery. As the signal travels from satellite to an earth station (or a mobile node) it undergoes a variety of 
impairments or losses. It can be safely assumed that the existing satellite link takes care of all such losses, except atmospheric attenuation (due to rain, ice, etc) and mobile channel losses (multipath fading and signal shadowing). These are closely related to the environment of the recipient $[21,27]$.

\section{B. Channel Model}

The most commonly used land mobile satellite channel (LMSC) model is a two-state Markov chain based channel model, which has been represented by a digital two-state Gilbert-Elliott model [21]. In this paper we will use this twostate channel model.

It is a two-state ON/OFF model. In ON (1) state the communication is error free after applying existing satellite communication channel coding; the state mainly covers line of sight (LOS) region. In OFF (0) state communication errors are beyond the existing channel correction capability and reliable communication is not possible, the state mainly covers non line of sight (NLOS)/shadowed/deep-fade regions [22]. Transition probabilities of this model depend on the environment (mean duration of ON/OFF state), vehicle speed and transmission (bit) rate [22]. The model excludes fading events with short durations, so the state transitions can be assumed to take place at cell boundaries, where a cell corresponds to a data segment. The average sojourn time in each state mainly depends on the environment in which the vehicle/node is moving.

\section{Satellite Communication}

The satellite downlink communication makes use of existing error correction techniques on each transmitted segment. It is assumed that the existing error correction techniques applied are sufficient to provide error free communication in the absence of deep fading and shadowing [22]. To further reduce the effects of segment loss due to deep fading and shadowing, time diversity is applied [23]. It can be achieved by inter-user or intra-user segment interleaving or both (Fig. 1). This helps in spreading the error among different users or different sessions, and by employing error correction techniques at higher layer the chances of recovery are improved. The interleaving removes the impact of consecutive losses and therefore we can assume that consecutive data segments of a session/user are independent of each other. In the rest of the paper (especially in figures) the segments considered/shown adjacent to each other are consecutive segments of a session and are not necessarily transmitted consecutively unless described/shown otherwise.

\section{Proposed Architecture}

In this paper our focus is on provision/extension of Internet to vehicular networks in rural areas and along the highways especially during the initial deployment stages. The working environment is characterized by a very small number of RSUs that are widely interspaced. These RSUs may be co-located with isolated populated areas along the highways and are connected to the Internet. The environment does not exhibit high shadow losses.

\section{A. Assumptions}

Our system design is based on a few simple assumptions. First, vehicles are equipped with GPS, can record their location at precise time and can provide direction of travel information to the RSU. Second, vehicles can receive the satellite broadcast. And third, RSUs have the digital map of the area and are aware of the locations of adjacent RSUs.

\section{B. Basic Idea}

A vehicle connects to a nearby RSU and requests some Internet data. The request will include location, speed and direction of travel of the vehicle. This information will help an RSU to calculate possible connection time left and possible next RSU. If sufficient connection time is left then the request may be serviced through the same RSU. When the vehicle exits the coverage of current RSU, further responses to the vehicle's earlier request will be sent to the next RSU in the direction of travel. If the two RSUs are located at a reasonable distance, which is likely in urban environment, the next RSU will continue to deliver the content to the vehicle when the vehicle comes within its coverage area (RSU-based region in Fig. 2). If the next RSU is not within a reasonable distance (especially in rural environment where the RSUs will be widely spaced) then satellite downlink channel will be used for delivery of content (satellite downlink-only region in Fig. 2). We will mostly address the satellite downlink option in this paper.

\begin{tabular}{|c|c|c|c|c|c|c|c|c|}
\hline$\left(\mathrm{U}_{1}, \mathrm{~F}_{1}, \mathrm{P}_{1}\right)$ & $\left(U_{1}, F_{1}, P_{2}\right)$ & $\ldots$ & $\left(U_{1}, F_{1}, P_{n}\right)$ & $\left(\mathrm{U}_{1}, \mathrm{~F}_{2}, \mathrm{P}_{1}\right) .$. & \multicolumn{2}{|c|}{\begin{tabular}{|l|l|}
$\left(U_{1}, F_{2}, P_{n}\right)$ & $\ldots$
\end{tabular}} & \multicolumn{2}{|c|}{$\left(U_{1}, F_{n}, P_{n}\right)$} \\
\hline \multicolumn{9}{|c|}{$\begin{array}{c}\text { Actual segment transmission sequence in time } \\
\mathrm{t}\end{array}$} \\
\hline$\left(\mathrm{U}_{1}, \mathrm{~F}_{1}, \mathrm{P}_{1}\right)$ & $\left(\mathrm{U}_{2}, \mathrm{~F}_{1}, \mathrm{P}_{1}\right)$ & $\ldots$ & $\left(U_{n}, F_{1}, P_{1}\right)$ & $\left(U_{1}, F_{1}, P_{2}\right)$ & $\ldots$ & $\left(U_{n}, F_{1}, P_{n}\right)$ & $\ldots$ & $\left(U_{n}, F_{n}, P_{n}\right)$ \\
\hline
\end{tabular}

Figure 1. Inter and Intra user segment interleaving to achieve time diversity.

While a vehicle travels between two RSUs in the satellite downlink-only region, it cannot send acknowledgements. In order to keep TCP connection alive and avoid unnecessary retransmissions, adaptive TCP timeout and delayed ACK will be used $[25,26]$. TCP timeouts will be calculated/predicted depending on the location of the next RSU and will be used accordingly. The downlink has large delays so in order to avoid unnecessary retransmissions selective acknowledgement will be used. Modified TCP is only employed between the proxy and mobile host so no modifications are required in protocols running on existing Internet. The use of UDP is much simpler than TCP and will not require any modifications.

The flow of traffic between different entities is outlined below (refer to Fig. 2):

- The mobile node authenticates with the proxy through an RSU and is issued with an IP address; this IP address will uniquely identify the mobile node as long as it remains within the boundary of the proxy.

- The mobile node sends a request to the RSU.

- The RSU acts as a router and forwards the request to the proxy.

- The proxy establishes a connection to Server on behalf 
of the mobile node and gets/caches all the content (based on initial request of the mobile node).

- The proxy establishes a connection with the mobile node on behalf of Server and sends the content via an RSU.

- When the mobile node moves out of the range of an RSU and the direct connection with RSU times out (Fig. 2, satellite downlink-only region); the RSU informs the proxy about expected time of next ACK from the mobile node. The time period depends on the speed and location of the next RSU along the travel direction of the mobile node.

- $\quad$ The proxy starts sending further content via satellite. It keeps on sending without waiting for ACK from the node till the time period expires.

- The mobile node keeps on receiving data via satellite till it reaches the next RSU (Fig. 2, end of satellite downlink-only region).

- The mobile node sends ACK for the received data or NACK for segments lost due to errors.

- $\quad$ The proxy updates the mobile node's new position and acts according to ACK/NACK.

- The process is repeated till all the requested content is delivered to the mobile node.

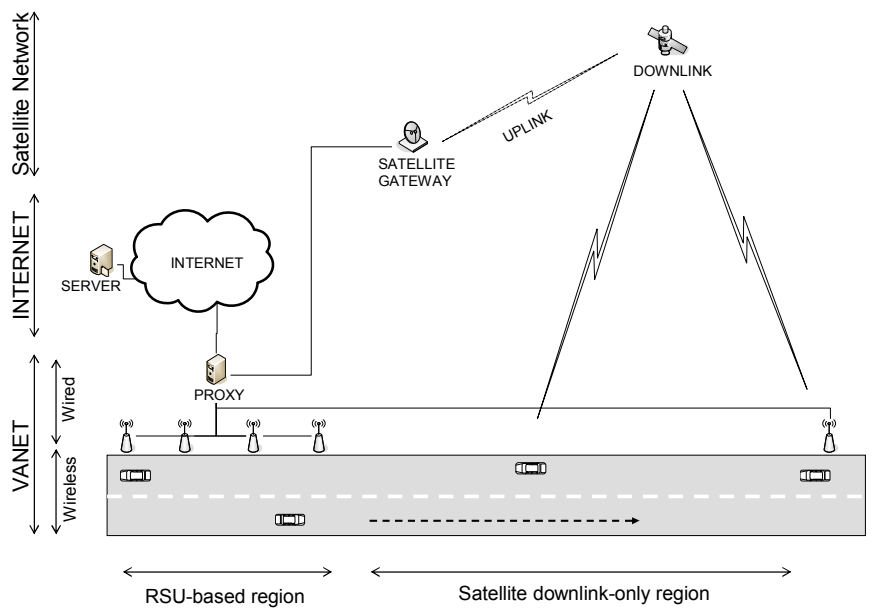

Figure 2. Proposed design in context of higher level vehicular network architecture.

1) Baseline Architecture: A vehicle sends a request to its nearby RSU (Fig. 3, $\mathrm{R}_{1}$ ), which in turn forwards the request to the proxy server. The proxy server gets the response/data from the server and forwards it to the satellite gateway. The proxy server splits the end-to-end connection between the vehicle and server [24]. It maintains two separate connections, one with the server on behalf of the vehicle and the other with the vehicle. The session with vehicle will be asymmetric, that is, the down link will be through satellite and return will be through RSUs. No modifications are required on the server side nor on the satellite downlink.

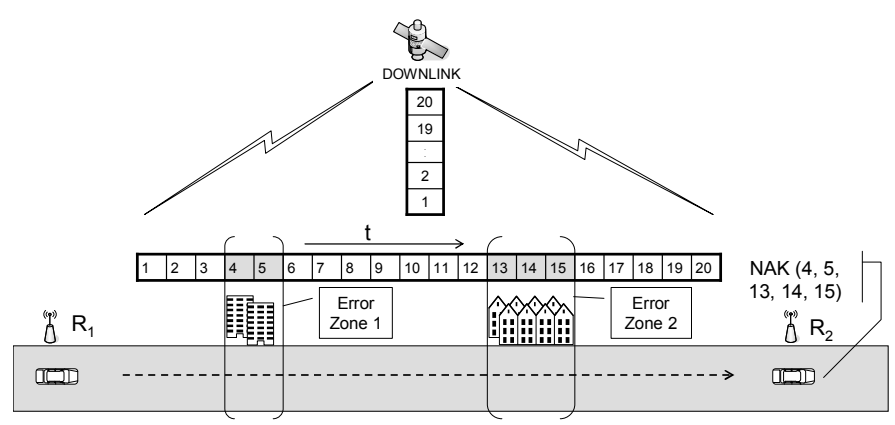

Figure 3. Baseline architecture, delivery of content takes place through satellite while the mobile node is traveling between $R_{1}$ and $R_{2}$. The mobile node sends NAK for the lost segments when reaching $R_{2}$, where $R_{2}$ takes charge and resends these lost segments to the mobile node.

The connection between the proxy and the vehicle will employ TCP enhancements/modifications such as Adaptive timeout, delayed ACK, and selective ACK/NAK $[25,26]$. The adaptive timeout caters for the time during which the vehicle cannot send ACKs, that is while traveling between the RSUs (Fig. 3, between $\mathrm{R}_{1}$ and $\mathrm{R}_{2}$ ).

When a request is received by the proxy, it forwards the request to the original server in a separate connection. It also calculates the timeouts and delays (for delayed ACK) expected based on the distance between adjacent RSUs (Fig. 3, between $\mathrm{R}_{1}$ and $\mathrm{R}_{2}$ ), the vehicle speed and its direction of travel. The proxy server receives all the data, which maybe a large file, from the server and also keeps the connection alive for further requests from the vehicle (this will be necessary if the transaction has to be completed after receiving some response from the vehicle during its connection with the next RSU). The proxy server then forwards the data to the vehicle through the satellite gateway and waits for the ACK/NAK. Because of high bandwidth-delay product (the delay of the satellite communication and also between sending the data and receiving ACK due to separation of RSUs), it may be necessary that all data segments are sent before waiting for an ACK/NAK from the vehicle.

If some of the received frames have been lost (Fig. 3, segments 4, 5, 13, 14 and 15) then the vehicle sends selective ACK/NAK on its next contact with roadside infrastructure (Fig. 3, $\mathrm{R}_{2}$ ). These ACK/NAK segments are forwarded to the proxy server, which retransmits the lost segments through satellite/RSU.

2) Repeated Transmissions: A method to address segment losses is by repeating the complete transmission in cyclic manner for a fixed number of times. This option adds maximum data redundancy. Although this is not an efficient utilization of the bandwidth available and we will have low information per bit transmitted, this approach can mitigate the effects of channel impairments. Especially during the initial VANET deployment stages when not many of smart vehicles will be on roads, a given satellite channel will be shared by a limited number of vehicles and each vehicle will have sufficient share of satellite bandwidth, which can be used for repeated transmissions. Also, during the initial stages there will be fewer number of RSUs which means larger distances 
between RSUs and more time to service a given request. This available time can be utilized for the redundancy.

This scheme suffers from long delays because in worst case a vehicle might have to wait for a complete cycle of retransmission to recover the lost data segment. It has high delay but is a suitable scheme when we are experiencing high error rate that cannot be corrected by other schemes.

Fig. 4 shows a vehicle driving between two widely spaced RSUs. The data is being sent through satellite which comprises of segments numbered 1 to 8 . The vehicle fails to receive segments 4 and 5 during the first transmission cycle since it was passing through error zone 1 during their transmissions. The vehicle recovers the lost segments from the second transmission cycle and is successful in receiving all eight segments before reaching the next RSU, where it acknowledges the receipt of all the segments.

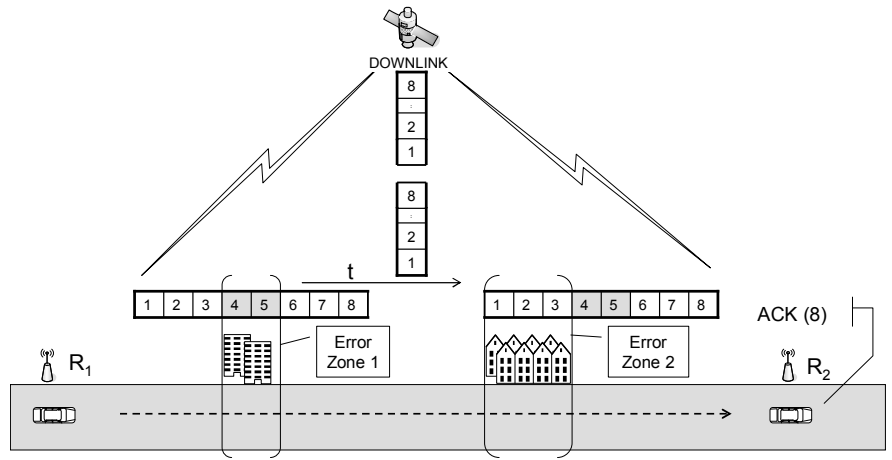

Figure 4. Repeated transmission, whole data set is repeated several times. Data segment(s) lost can be recovered from later repeated transmissions.

3) Error Location Prediction and Avoidance: The satellite mobile channel suffers mostly from shadowing and fading. These errors are strongly correlated to the environment. Vehicles traveling along a particular highway are expected to experience channel impairments at approximately the same locations (refered as "error zones"). If the location of these error zones can be registered and the segments that were sent to a vehicle while it was passing through these error zones can be determined, then these segments may be retransmitted to the vehicle without waiting for an ACK/NAK from the vehicle. This will improve the performance since the vehicle does not need to wait for the next RSU, which may be quite far off, to recover from the error.

The location of error zones can be determined if a vehicle also includes location information with NAK, which is the location where segment loss was experienced. This location information is used to predict the possible location of segment losses for future vehicles traveling along the same path. For example in Fig. 3 the vehicle experiences loss of segments 4 and 5 in error zone 1 and can report $\{$ NAK $(4,5)$, Error Zone 1$\}$

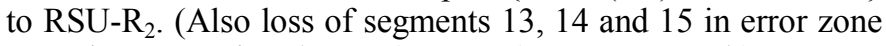
2 can be reported as $\{\operatorname{NAK}(13,14,15)$, Error Zone 2$\})$.

The time period during which the vehicle was traveling through a registered error zone can be estimated from the vehicle's speed and its initial time-location information. The fact that the speed of vehicles has generally less variations in highway environment also helps in minimizing the estimation error. However, an error margin can be added on both sides of probable error location to cater for variations in driving speeds. The RSU with which the vehicle was last authenticated/associated (Fig. 5, $\mathrm{R}_{1}$ ) listens for the segments which were sent during the error zone period and sends NAK to the proxy for retransmission of these segments.

Take Fig. 5 as an example. A vehicle sends a request to RSU $R_{1}$, which forwards the request to the proxy server and data is sent to the vehicle via satellite. The RSU $\mathrm{R}_{1}$ calculates approximate times $\left(\left\{\mathrm{t}_{0}-\mathrm{t}_{1}\right\}\right.$ and $\left.\left\{\mathrm{t}_{2}-\mathrm{t}_{3}\right\}\right)$ when the vehicle will be passing through error zones. It then monitors the satellite transmissions destined to this vehicle. It records the segment numbers sent by satellite when the vehicle passes through these error zones (segments $\{4,5\},\{11,12,13\}$ ) and sends NAK to the proxy server for these segments (segments NAK $\{4,5\}$, NAK $\{11,12,13\})$. When receiving these NAKs, The proxy server retransmits the lost segments through satellite.

When the vehicle reaches the next RSU, it sends NAK if it still could not receive all the segments. These NAK locations are then used by previous RSU to modify its error zone information. It is important to note that there is no way for the initial RSU to know if an earlier reported error zone has disappeared or not. There may be a situation (e.g. heavy rain) when most of the locations are marked as error zones. To address this possible situation it is necessary from time to time to reset the error zones to zero.

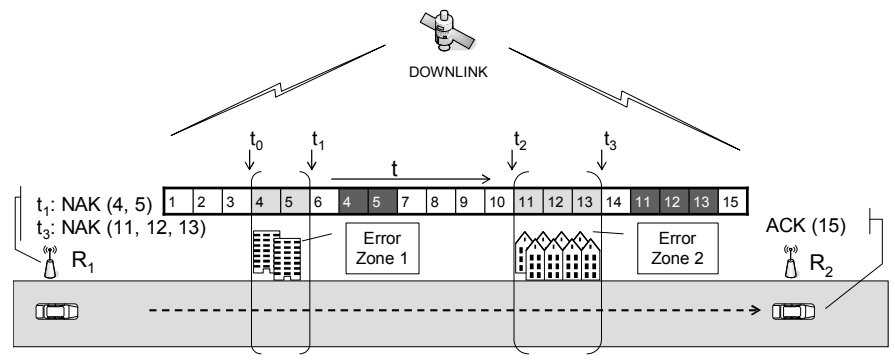

Figure 5. Error location prediction and avoidance. The system predicts the segments which may have been lost on the bases of previous data and proactively retransmits these segments.

\section{Comparison of Options}

The options presented in preceding sections offer different levels of error tolerance at the cost of overheads and delays. One must balance the performance (error tolerance) vs. cost (overheads and delays) in selecting a particular solution; also, some options may be more suited to a particular environment than the other environments. The baseline architecture uses simple ACK/NAK for flow control and error correction. This scheme has no overhead but successful completion of communication may be delayed till the vehicle reaches the next RSU. This architecture is suitable where RSUs are not very widely dispersed. Error location prediction and avoidance uses proactive retransmission of predicted lost segments. This scheme has low overheads and low delays. This scheme is especially useful where satellite mobile channel losses are reasonably localized in certain areas. Repeated Transmission is the most robust scheme, it is especially useful where longer 
durations of Bad state is experienced, but at the cost of having high overhead and medium delays. A summary of the options with recommended usage is also presented in Table I.

TABLE I. RECOMMENDED USAGE OF DIFFERENT OPTIONS

\begin{tabular}{|l|l|l|l|}
\hline \multicolumn{1}{|c|}{ Option } & Overhead & Delay & \multicolumn{1}{|c|}{ Recommended Usage } \\
\hline Baseline & None & High & $\begin{array}{l}\text { Where RSUs are not very } \\
\text { widely spaced }\end{array}$ \\
\hline $\begin{array}{l}\text { Repeated } \\
\text { Transmission }\end{array}$ & High & Medium & $\begin{array}{l}\text { Where Bad state duration is } \\
\text { longer than FEC can tolerate }\end{array}$ \\
\hline $\begin{array}{l}\text { Error Location } \\
\begin{array}{l}\text { Prediction and } \\
\text { Avoidance }\end{array}\end{array}$ & Low & Low & $\begin{array}{l}\text { Where Bad state } \\
\text { environments are relatively } \\
\text { stable over a relatively long } \\
\text { period of time }\end{array}$ \\
\hline
\end{tabular}

\section{CONCLUSION AND FUTURE WORK}

We have presented a viable solution for provision of the Internet access to the vehicular networks, especially during the initial deployment phase of vehicular networks and also in areas with very scarce roadside infrastructure (such as along highways and in rural areas). The solution is practical and economical since it only uses satellite receive-only terminals and very few (widely spaced) RSUs. We have also presented a number of error handling options which can be employed according to the operating environments.

The efficiency of the solution can be further enhanced by using V2V communication in a variety of ways. For example, caching and later relaying the data for other vehicles (that might not have been able to receive it due to error zone), relaying NAK to previous RSU (via vehicles traveling in opposite direction), using $\mathrm{V} 2 \mathrm{~V}$ communication as the reverse channel to send all the selective ACKs and NAKs, etc.

The solution is best suited for request-response type of applications, where a small request is followed by a large response data (such as file transfer, multimedia download, etc). The solution does not provide continuous connectivity so interactive or continuous connectivity demanding applications, such as IP telephony cannot be supported. Also, the solution is not intended to support security based applications that are time critical and require large data flow from vehicles; however, non-time critical or broadcast nature of security applications are supported, for example, dissemination of certificate revocation lists (or other security alerts) through satellites.

\section{REFERENCES}

[1] M Bechler, WJ Franz, and L Wolf, "Mobile internet access in FleetNet", 13th Fachtagung Kommunikation in verteilten Systemen, Leipzig, April 2003.

[2] Franz, W. and Hartenstein, H. and Bochow, B., "Internet on the road via inter-vehicle communications", in GI/OCG Annual Conference: Workshop on Mobile Communications over Wireless LAN: Research and Applications, Vienna, Sep 2001.

[3] J. Ott, and D. Kutscher, "Drive-thru Internet: IEEE $802.11 \mathrm{~b}$ for automobile users", in IEEE Infocom Conf., 2004.

[4] Y. Yang, M. Marina, and R. Bagrodia, "Evaluation of multihop relaying for robust vehicular Internet zccess", in MObile Networking for Vehicular Environments (MOVE), 2007.
[5] V. Bychkovsky, B. Hull, A. Miu, H. Balakrishnan, and Samuel Madden, "A measurement study of vehicular internet access using in situ Wi-Fi networks", in 12th annual intl. conf. on Mobile computing and networking, Los Angeles, USA, Sep 2006.

[6] R. A. Wyatt-Millington, R Sheriff, Y. F. Hu, P. Conforto, and G. Losquadro, "The SUITED project: a multi-segment system for broadband access to Internet services", in IEE Broadband Satellite Conf., London, England, 2000.

[7] J. Santa, R. T. Moreo, and A. F. G. Skarmeta, "A novel vehicle communication paradigm based on cellular networks for improving the safety in roads", in Int. J. Intelligent Information and Database Systems, Vol. 2, No. 2, pp. 240-257, 2008.

[8] D.G. Oh, P. Kim, Y.J. Song, I.J. Jeon, and H.-J. Lee, "Design considerations of satellite-based vehicular broadband networks", in IEEE Wireless Comm., vol. 12, Oct 2005, pp. 28-36.

[9] J. L. Mineweaser, J. S. Stadler, S. Tsao and M. Flanagan, "Improving TCP/IP performance for the land mobile satellite channel", in IEEE Military Comm. Conf., 2001.

[10] T. T. Kwan , R. E. McGrath , D. A. Reed, "User access patterns to NCSA"s World Wide Web server", University of Illinois at UrbanaChampaign, Champaign, IL, 1995.

[11] G. Fairhurst, N.K.G. Samaraweera, M. Sooriyabanadara, H. Harun, K. Hodson, and R. Donadio, "Performance issues in asymmetric TCP service provision using broadband satellite", in IEE Comm, Vol 148 No 2, April 2001.

[12] I. Minei and R. Cohen, "High-speed Internal access through unidirectional geostationary satellite channels, in IEEE JSAC, vol. 17, no. 2, pp. 345-359, Feb. 1999.

[13] J. Gutiérrez. "Selected readings on telecommunications and networking", Idea Group Inc (IGI), 2008.

[14] J. Luo and J.-P. Hubaux, "A survey of research in inter-vehicle communications", in Securing Current and Future Automotive IT Applications, pp 111-122, Springer-Verlag, 2005.

[15] R. Chakravorty, A. Clark and I. Pratt, "GPRSWeb: optimizing the web for GPRS Links", in ACM/USENIX MobiSys, San Francisco, May 2003

[16] M. C. Chan and R. Ramjee, "TCP/IP performance over $3 \mathrm{G}$ wireless links with rate and delay variation", in ACM Mobicom, Sep 2002.

[17] G. Giambene and D. Miorandi, "A simulation study of scalable TCP and highSpeed TCP in geostationary satellite networks", in J. Telecomm systems, Vol 30, No 4, pp. 297-320, Dec 2005.

[18] P. Papadimitriou, and V. Tsaoussidis, "On TCP performance over asymmetric satellite links with real-time constraints", in Jour. Computer Comm., Vol 30 No 7, pp. 1451-1465, May 2007.

[19] G. Giambene and M. Marandola, "Internet access in hybrid terrestrial and satellite mobile communication systems", in IEEE VTC, 2004.

[20] T. A. Gillespie, "Modeling the transformational communications system urban land mobile satellite channel", 2007, Storming Media, http://www.stormingmedia.us/14/1406/A140674.pdf.

[21] E. Lutz, M. Werner, and A. Jahn, "Satellite systems for personal and broadband communications", Berlin, Germany: Springer-Verlag, 2000.

[22] S. Scalise,H. Ernst, and G. Harles, "Measurement and modeling of the land mobile satellite channel at Ku-band," in IEEE Trans. Veh. Tech., Vol 57, No 2, pp. 693-703, March 2008.

[23] C. Martin, A. Geurtz, and B. Ottersten, "Statistical analysis and optimal design for efficient mobile satellite broadcast with diversity", in IEEE Trans. on Veh. Tech., Vol 57, No 2, pp 986-1000, March 2008.

[24] J. Border, M. Kojo, J. Griner, G. Montenegro, and Z. Shelby, "RFC 3135: Performance enhancing proxies intended to mitigate link-related degradations', IETF RFC 3135, June 2001.

[25] M. Mathis, J. Mahdavi, S. Floyd, and A. Romanow, "TCP selective acknowledgement options", IETF RFC 2018, October 1996.

[26] L. Eggert and F. Gont, "TCP user timeout option, standards track (draftietf-tcpm-tcp-uto-11), January 22, 2009. 